\title{
Psychische Ereignisse - organische Interpretationen: Traumakonzepte in der deutschen Psychiatrie seit 1889
}

Ruth Kloocke, Heinz-Peter Schmiedebach und Stefan Priebe

\section{Summary}

This paper examines the debate on psychological trauma in German psychiatry since 1889 . A content analysis of five leading German psychiatric journals between 1889 and 2005 is realised. An organic concept of psychological trauma has been prominent in the professional debate until today. Psychiatrists frequently referred to physical traumatisation, constitutional factors and genetic predisposition, exogenous reaction types according to Bonhoeffer, and to biological markers in the context of the PTSD concept. The biological tradition in German psychiatry resulted in a specific adoption of concepts on psychological trauma. However, integrating various models of psychological trauma into a psychiatric tradition focusing on a biological model proved to be difficult and inconsistent.

Keywords: psychological trauma; organic interpretation; academic psychiatry; German history

\section{Zusammenfassung}

Dieser Aufsatz untersucht die psychiatrische Fachdiskussion zu psychischer Traumatisierung in Deutschland seit 1889. Wir identifizieren dominierende Begriffe und Konzepte, mit denen psychiatrische Experten das Phänomen

Dr. Ruth Kloocke, Unit for Social and Community Psychiatry, Newham Centre for Mental Health, UK-London E13 8SP (ruth.kloocke@beh-mht.nhs.uk).

Prof. Heinz-Peter Schmiedebach, Institut für Geschichte und Ethik der Medizin, Universitätsklinikum Hamburg-Eppendorf, Martinistrasse 52, D-20246 Hamburg (p.schmiedebach@uke. uni-hamburg.de).

Stefan Priebe, Klinik für Psychiatrie und Psychotherapie, Charité Campus Mitte, Universitätsmedizin Berlin, Schumannstrasse 20/21, D-10117 Berlin (s.priebe@qmul.ac.uk). 
des psychischen Traumas zu beschreiben versuchten, sowie deren Wandel im Verlauf von 117 Jahren. Inhaltliche Analyse der Veröffentlichungen und Diskussionsbeiträge zu psychischer Traumatisierung werden in fünf führenden deutschen Fachzeitschriften für Psychiatrie von 1889 bis 2005 aufgeführt. Wir finden, dass eine organische Konzeptionalisierung von psychischer Traumatisierung bis in die Gegenwart dominant ist. Organische Konzepte, auf die häufig referiert wurde, waren: postulierte physische Traumatisierung, Konstitution im Sinne einer genetischen Anlage, die exogenen Reaktionstypen Bonhoeffers und biologische Faktoren und Marker im Rahmen des PTSDKonzeptes. Die biologische Tradition in der deutschen Psychiatrie führte zu einer spezifischen Rezeption von Traumakonzepten, nicht aber zu einer Negierung des psychischen Traumas per se. Die Einbindung des psychischen Traumas in das naturwissenschaftliche Modell der akademischen Psychiatrie erwies sich als schwierig und widersprüchlich.

\section{Einleitung}

Das Thema «psychisches Trauma» hat in der deutschen Psychiatrie eine lange Tradition. In der vorliegenden Arbeit versuchen wir, der theoretischen Debatte zu psychischer Traumatisierung systematisch nachzugehen, diese im Zusammenhang darzustellen und aufzuzeigen, in welchen Begriffen, Konzepten und Kontexten innerhalb der deutschen akademischen Psychiatrie über psychisches Trauma nachgedacht wurde. Unser Ziel ist, die verschiedenen, oft parallel verlaufenden und manchmal auch widersprüchlichen Entwicklungen im Überblick darstellen. Wir können dabei auf die Ergebnisse einer Reihe von zum Teil detaillierten medizinhistorischen Arbeiten zur Geschichte des psychischen Traumas in der deutschen Psychiatrie zurückgreifen ${ }^{1}$. Die meisten dieser Untersuchungen beziehen sich auf den Zeitraum bis zum Ende des 2. Weltkriegs. Pross, Peters und Goltermann untersuchen bestimmte Aspekte der Traumadebatte der Nachkriegszeit, während ansonsten wenige Arbeiten zum Zeitraum nach 1945 vorliegen.

1 Fischer-Homberger 1975; Roth 1987; Pross 1988; Peters 1989; Eghigian 1991; Komo 1992; Hilpert 1995; Riedesser/Verderber 1996; Berger 1998; Lembach 1998; Fischer-Homberger 1999; Blaßneck 2000; Micale/Lerner 2001; Müller 2001; Goltermann 2002; Lerner 2003; Thomann/Rauschmann 2003; Tölle 2005. 


\section{Fragestellung}

Diese Arbeit untersucht die professionelle Debatte bezüglich psychischer Traumatisierung in der deutschen akademischen Psychiatrie zwischen 1889 und 2005. Sie möchte die Grundlage für ein Verständnis der Entwicklung der Krankheitskonzepte zu psychischer Traumatisierung bis in die Gegenwart schaffen. Wesentliche Fragestellungen unserer Untersuchung lauten: Welche Art von Ereignissen wurde von psychiatrischen Experten zu verschiedenen Zeiten als psychisches Trauma wahrgenommen? Welche Symptome oder Syndrome wurden im Zusammenhang mit diesen Traumen geschildert? Welche Begriffe ordneten psychiatrische Experten diesen Phänomenen zu? Welche Krankheitsmodelle standen hinter den jeweiligen Begriffen?

\section{Methode}

Im Rahmen eines DFG-Projektes zur Geschichte des psychiatrischen Traumabegriffs ( $\operatorname{Pr} 437 / 6-1$ und $\operatorname{Pr} 437 / 6-2$ ) untersuchten wir in einer Kooperation von Psychiatern und Medizinhistorikern Veröffentlichungen zum Thema «psychisches Trauma» in fünf führenden deutschen Zeitschriften für Psychiatrie. Herangezogen wurden Allgemeine Zeitschrift für Psychiatrie und psychisch-gerichtliche Medizin; Archiv für Psychiatrie und Nervenkrankheiten; Der Nervenarzt; Psychiatrie, Neurologie und medizinische Psychologie sowie Fortschritte der Neurologie, Psychiatrie und ihrer Grenzgebiete. Auswahlkriterium war, dass die Zeitschriften wichtige Fachgesellschaften repräsentierten und über einen langen Zeitraum der gewählten Untersuchungsperiode hinweg erschienen. Diese Quellengrundlage ermöglichte auch einen Vergleich zwischen Ost- und Westdeutschland für die Zeit der deutsch-deutschen Teilung. Die Zeitschrift Fortschritte der Neurologie, Psychiatrie und ihrer Grenzgebiete wurde einbezogen, da sie insbesondere in den ersten Jahrzehnten ihres Bestehens das erklärte Ziel hatte, den aktuellen Stand der Forschung in Überblicksreferaten darzustellen. Als Ausgangspunkt wählten wir mit 1889 das Jahr der Veröffentlichung von Hermann Oppenheims Schrift Die traumatischen Neurosen ${ }^{2}$. Alle zwischen 1889 und 2005 erschienen Jahrgänge dieser fünf Zeitschriften, mit Ausnahme von sechs nicht verfügbaren Bänden, wurden ausgewertet. Ergänzend wurden weitere in den Zeitschriften zitierte Veröffentlichungen herangezogen.

2 Oppenheim 1889. 
Wir unterteilten den Untersuchungszeitraum pragmatisch in Zeitabschnitte von rund 25 Jahren Dauer, welche mit wichtigen historischen Einschnitten koinzidieren: (a) die Zeit vor dem ersten Weltkrieg 1889-1914, (b) die Zeit des 1. Weltkriegs und die Zwischenkriegszeit 1914-1938, (c) die Zeit des 2. Weltkriegs und die Nachkriegszeit 1939-1964, (d) die Zeit von 1965 bis 1989, (e) die jüngere Vergangenheit nach der deutschen Wiedervereinigung 1990-2005.

Bei der Auswahl der Beiträge wurde abweichend von den gegenwärtig dominierenden operationalisierten Kriterien eine weite, alle Einwirkungen von gravierenden äusseren Ereignissen einschliessende Definition von psychischem Trauma verwendet, um auch die Grenzen der Traumadebatte abzudecken, die sich im Laufe der Zeit wiederholt verschoben. In einer Diskussion der Arbeitsgruppe wurde eine Liste von Stichwörtern entwickelt, um Beiträge zur Traumadebatte zu identifizieren. Als Stichwörter benutzten wir: Trauma, traumatische Neurose, traumatische Psychose, Unfall, Unfallneurose, Krieg, Kriegsneurose, Kriegspsychose, Rentenneurose, Haft, Haftneurose, Haftpsychose, Ganser-Syndrom, Reaktion, reaktiv, abnorme Reaktion, psychogene Reaktion, psychopathische Reaktion, Psychopathie, KZ-Syndrom, Überlebenden-Syndrom, erlebnisbedingter Persönlichkeitswandel, Anpassungsstörung, PTSD, Posttraumatische Belastungsstörung, Stress, Erschöpfung, Vergewaltigung, Naturkatastrophe, Erlebnis, Begutachtung, Entschädigung, Kausalität und Ätiologie³ .

\section{Ergebnisse}

Wir identifizierten in unseren Quellen rund 550 Beiträge zum Thema «psychisches Trauma». Der Grossteil (>80\%) dieser Beiträge wurde bereits bis Mitte der 1960er Jahre veröffentlicht, während das Interesse am Thema in der zweiten Hälfte des 20. Jahrhunderts abzuflauen schien. Auf dem Höhepunkt der Debatte im Jahr 1916 fanden wir 24 Veröffentlichungen zum Thema. Ein aus heutiger Perspektive überraschendes Ergebnis unserer Untersuchung war, dass sich ein substantieller Teil dieser Beiträge (rund 200) mit dem Thema «Trauma und Psychose» befasste. Wir haben dementsprechend dem Thema «Trauma und Psychose» eine spezifische Untersuchung gewidmet ${ }^{4}$.

3 Die Begriffe «Schock» und «shellshock», die in einer Analyse der englischsprachigen Lehrbücher für Psychiatrie nach 1945 regelhaft auftauchten, spielten interessanterweise in unseren Quellen für die vorliegende Untersuchung keine Rolle (vgl. Wildgrube et al. 2010).

4 Kloocke et al. 2010. 
Von 1889 bis zum Ausbruch des ersten Weltkrieges fanden wir 96 Originalien, Mitteilungen und Diskussionsbeiträge zum Thema. Häufig verwendete diagnostische Kategorien sind die Unfallhysterie, Unfallneurose, hysterische Unfallerkrankungen, Nervenerkrankungen nach Unfall, psychogene Krankheitszustände, traumatische Psychose, Erschöpfungspsychose, hysterischer Dämmerzustand, Ganser'sches Syndrom, hysterischer Stupor, Gefängnispsychose, Unfallpsychose, hysterisches Irresein, Haftpsychose, Situationspsychose der Kriminellen und hysterische Situationspsychose. Oft ist erst aus dem Kontext zu ersehen, dass diese diagnostischen Kategorien in Zusammenhang mit einem auslösenden äusseren Ereignis - einem plötzlichen psychischen Trauma oder einer gravierenden, anhaltend belastenden Situation - diskutiert wurden. Die am häufigsten genannten auslösenden Ereignisse dieser Periode sind Unfälle und Haft.

Psychiatrische Experten um die Wende zum 20. Jahrhundert interessierten sich sehr für die Frage, ob psychische Traumen Psychosen auslösen kön$n^{5}{ }^{5}$. Unterschiedliche psychiatrische Konzeptionen gingen in diese Diskussion ein. Eine klare Trennung zwischen Psychosen und Neurosen gibt es zu dieser Zeit noch nicht. Um 1900 wurden post-traumatische Erkrankungen häufig in den Kontext des hysterischen Spektrums gestellt. Ein wesentlicher Aspekt dieses Krankheitsspektrums war die Vielfalt der Symptome, zu der in dieser Zeit durchaus auch psychotische Symptome gerechnet wurden ${ }^{6}$. Analog zu dem so genannten hysterischen Irresein wurde auch vom traumatischen Irresein gesprochen.

Besonders wichtig in der Diskussion um psychische Traumen wurden nach 1900 Fragen der Forensik. Eine Vielzahl von Publikationen beschäftigt sich mit den Haftpsychosen oder Gefängnispsychosen, zu denen auch die Ganser'schen Dämmerzustände gerechnet wurden ${ }^{7}$. Diese Thematik gab der Diskussion eine neue Wendung, indem sie eine Verknüpfung mit Fragen der Vererbungsforschung und der Degenerations- und Entartungslehre herstellte. Die Autoren diskutierten, ob es eine spezifische Gefängnispsychose gebe. Die Mehrheit der Experten, wie zum Beispiel Kurt Wilmanns und Felix Stern, grenzte nach der Jahrhundertwende in der Haft zu beobachtende Fälle von

5 Von 96 Beträgen 48: z.B. Richter 1891; Edel 1901; Wende 1904; Weber 1905 oder HascheKlünder 1908.

6 Hess 1903; Raecke 1905, 171.

7 Seifert 1900; Raecke 1901; Rüdin 1901; Rüdin 1903; Bumke/Rosenfeld 1910; Wilmanns 1910; Heinecke 1911; Anonym 1912; Stern 1913 und Anonym 1913. Vgl. zur Geschichte des Ganser'schen Syndroms auch Kloocke et al. 2010. 
Dementia praecox deutlich von den Situationspsychosen oder degenerativen Haftpsychosen ab, die sie dem hysterischen Spektrum zuordneten ${ }^{8}$. August Homburger definierte diese als «pathologische Reaktionen abnormer Persönlichkeiten auf die Einflüsse des Strafvollzugs» und betonte deren gute Prognose: «Soweit sie nicht während der Strafzeit selbst abliefen, heilten sie nach der Entlassung in der Freiheit. Die Psychose ist im Lebenslauf dieser Leute lediglich eine für dessen fernere Gestaltung belangslose Episode» ${ }^{9}$. Die im Kontext der Forensik intensiv diskutierte Ätiologiefrage (Umgebungseinfluss versus Anlage) förderte bei vielen Autoren die Ansicht, für die traumatischen Psychosen eine degenerative Anlage zu postulieren. Nicht zuletzt trug die Verlagerung der Debatte um die traumatischen Psychosen in den Kontext der Haft mit zu einer «Kriminalisierung» der Thematik bei, was sich in der vehement geführten Debatte zur möglichen Simulation widerspiegelt.

Mit der Erschöpfungspsychose konzeptualisierte Binswanger oder Raecke ein weiteres Modell einer durch äussere Belastung verursachten Psychose, das stärker auf eine organische Ätiologie abzielte und beispielsweise Hunger oder Ernährungsstörungen als wichtige Faktoren betrachtete ${ }^{10}$. Die traumatischen Neurosen Oppenheims spielten in unseren Quellen allenfalls eine marginale Rolle. Der Tenor der Diskussionsbeiträge zur traumatischen Neurose, soweit sie überhaupt Erwähnung findet, ist ablehnend. Bereits 1890 initiierte Friedrich Schultze aus Bonn eine Diskussion zur Verwendung des Begriffs «traumatische Neurose», an der sich Edinger, Hitzig, Bäumler, Fürstner, Rumpf, Thomsen und Jolly beteiligten. In dieser Diskussion wurden die Schwierigkeiten der Abgrenzung der Diagnose betont. Die Mehrheit der Diskussionsteilnehmer stellte die Verlässlichkeit der von Oppenheim vorgeschlagenen organischen Stigmata (Beschleunigung der Pulsfrequenz, Sensibilitätsstörungen, Gesichtsfeldeinengung) in Frage. Ähnlich wie bei der späteren Debatte um die Haftpsychosen verwiesen viele Diskussionsteilnehmer auf die Häufigkeit der Simulation und damit auch auf die Fragwürdigkeit des Krankheitsbildes an sich.

Jolly schlug vor, den Begriff der traumatischen Neurosen ganz fallen zu lassen und entsprechende Krankheitsbilder als durch Traumata entstandene Hysterie zu benennen ${ }^{11}$. Häufiger als von der traumatischen Neurose wurde in dieser Zeit von den nervösen Unfallerkrankungen, den hysterischen Unfallerkrankungen, der Unfallhysterie oder der Unfallneurose gesprochen ${ }^{12}$. Der Terminus «traumatische Neurose» konnte sich jedoch halten und fand

8 Wilmanns 1908; Stern 1912; Stern 1913.

9 Bumke/Rosenfeld 1910, 811.

10 Grossmann/Schulze 1896; Raecke 1900.

11 Laquer/Buchholz 1890, 656.

12 Z.B. Seifert 1900; Bähmig 1903; Seifert 1903; Merzbacher 1907 und Winscheid 1908. 
auch internationale Verbreitung. Beispielsweise hielt 1912 der bekannte italienische Psychiater Augusto Murri in Mailand einen Vortrag über die traumatischen Neurosen, der auch ins Deutsche übersetzt wurde ${ }^{13}$.

\section{b) $1914-1938$}

Der Kriegsausbruch 1914 verschob das Zentrum der Diskussion von den Haft- und Unfallfolgen zu den Auswirkungen des Krieges. Literaturberichte in der Allgemeinen Zeitschrift vermitteln einen Eindruck von der Fülle der Publikationen zu diesem Thema ${ }^{14}$. Mit den Kriegsneurosen, die wenig später meist als Rentenneurosen apostrophiert wurden, rückte Oppenheims Krankheitsmodell wieder ins Interesse der psychiatrischen Fachdiskussion. Die erneute, vehemente Ablehnung der traumatischen Neurosen durch eine Mehrzahl der psychiatrischen Experten auf der Münchner Kriegstagung im Jahr 1916 erlebte Oppenheim als persönliche Niederlage ${ }^{15}$. Die in den folgenden Jahrzehnten erscheinenden Beiträge verwenden als diagnostische Kategorien fast ausschliesslich die Termini «Kriegsneurose», «Rentenneurose», «Unfallneurose», «Rechtsneurose» und «reaktive Neurose». Die Münchner Kriegstagung etablierte ein Krankheitsmodell, das zum Konsens unter den deutschen Psychiatern wurde: Psychoreaktive Symptome nach traumatischen Ereignissen wurden jetzt regelhaft als hysterische oder abnorme Reaktionen infolge einer psychopathischen Anlage eingeordnet und eine Entschädigung grundsätzlich abgelehnt. Die Psychiater der Kriegs- und Zwischenkriegszeit hatten damit die neurotischen Traumafolgen auf eine wirksame Formel gebracht, die klare Handlungsstrategien für Therapie und Prophylaxe und eine Entscheidungsgrundlage für die zentrale Entschädigungsfrage bereitstellte.

Die meisten Veröffentlichungen zur Rentenneurose oder Unfallneurose in den zwanziger und dreissiger Jahren beschäftigten sich mit der Anwendung dieses Krankheitsmodells auf Begutachtungsfragen und auf die praktische Bewältigung einer oft als überwältigend, frustrierend oder sogar moralisch entrüstend wahrgenommenen Aufgabe ${ }^{16}$. De facto betrug der Anteil der «Kriegsneurotiker» an allen Kriegsrentenanträgen hingegen lediglich $1-2 \%{ }^{17}$. Veröffentlichungen von Bonhoeffer und Stier bereiteten eine

13 Anonymus 1913.

14 Bresler 1919; Laehr 1919; Umpfenbach 1919a; Umpfenbach 1919b.

15 Fischer-Homberger 1975; Lerner 2003.

16 Moerchen 1928; Jossmann 1929; Stiefler 1929; Jossmann 1930; Riese 1930; Stiefler 1931; Abenheimer 1933 und Gruhle 1933.

17 Lerner 2003, 33. 
Grundsatzentscheidung des Reichsversicherungsamtes im Jahr 1926 vor, die eine Entschädigungspflichtigkeit von reaktiven Neurosen grundsätzlich ablehnt ${ }^{18}$. Diese Veröffentlichungen führten den Begriff der Krankheitsvorstellung in die Diskussion ein, der ausschlaggebend für die Ablehnung von Rentenansprüchen wurde: die «Idee, krank zu sein» wurde als nicht entschädigungsfähig erklärt ${ }^{19}$.

Die Diskussion zu den traumatischen Psychosen wurde nach 1914 mit den Begriffen «Kriegspsychose», «symptomatische Psychose», «Erschöpfungspsychose», «organischer Dämmerzustand», «hysterisches 〈Wunschdelirium»», «psychogene Psychose» und «reaktive Psychose» fortgeführt. Mit den so genannten exogenen Reaktionstypen formulierte Bonhoeffer ein organisches Paradigma für diese Art der Störungen, das in den kommenden Jahrzehnten die Diskussion weitgehend dominierte und auch in der Frage der Entschädigung handlungsrelevant wurde ${ }^{20}$. Kriegspsychosen wurden nach diesem Modell häufig organisch als durch Hunger, Erschöpfung oder Infektionskrankheiten bedingt interpretiert, sofern sie nicht als Aktivierungen von endogenen Psychosen angesehen wurden.

Im Gegensatz zu dieser Position stehend veröffentlichte Karl Kleist 1918 eine ausführliche Untersuchung zu den Schreckpsychosen, die er auch als psychogene Psychosen bezeichnete ${ }^{21}$. Kleist berichtete über 104 Fälle von im Durchschnitt 10 Tagen, gelegentlich länger anhaltenden Dämmer- oder Stuporzuständen, zum Teil in Kombination mit Halluzinationen auftretend, die er bei Kriegsteilnehmern im Stellungskrieg an der Westfront beobachtete. Er führte diese Zustände ausdrücklich nicht auf eine organische Hirnschädigung zurück und betonte, dass diese auch ohne psychopathische Veranlagung auftreten könnten ${ }^{22}$. Bonhoeffer sah die traumatische Ätiologie der Schreckpsychosen als nicht nachgewiesen an, und Kleists Position blieb letztlich wenig einflussreich ${ }^{23}$.

Parallel entwickelte sich um den ersten Weltkrieg herum eine breite Debatte, ob endogene Psychosen, insbesondere die Schizophrenien, durch psychische Traumen ausgelöst werden können. Sehr unterschiedliche Bezeichnungen wurden für diese auch als reaktive Psychosen zusammengefassten Bilder geprägt, so zum Beispiel schizophrene Reaktionen, funktionelle Schizophrenien, schizoforme Reaktionen, schizophrener Reaktionstypus, erleb-

18 Bonhoeffer 1926; Stier 1926.

19 Feilchenfeld 1927, 274.

20 Bonhoeffer 1908; Bonhoeffer 1910; Bonhoeffer 1917.

21 Kleist 1918a; Kleist 1918b.

22 Kleist 1918b, 484.

23 Bonhoeffer 1922, 8. 
nisreaktive Schizophrenien ${ }^{24}$. Payk konnte zeigen, dass sich diese Diskussion bis weit nach dem 2. Weltkrieg fortsetzte, obwohl Bonhoeffer es bereits 1922 als durch die Erfahrungen des 1. Weltkriegs erwiesen ansah, dass endogene Psychosen von aussen nur wenig oder gar nicht beeinflusst werden ${ }^{25}$.

\section{c) $1939-1964$}

Anders als im 1. Weltkrieg erschien während des 2 . Weltkriegs nur eine vergleichsweise kleine Zahl von Veröffentlichungen, die sich mit psychischen Traumen beschäftigten. Die Mehrzahl von diesen waren Übersichtsarbeiten, z.B. zur Psychopathie oder zu den psychogenen Reaktionen. Kein einziger Beitrag nahm im Titel direkt auf das Kriegsgeschehen Bezug. Für den Gesamtzeitraum des zweiten Weltkriegs und die beiden Jahrzehnte danach fanden wir hingegen 133 Publikationen. Psychisches Trauma wurde demnach in der Nachkriegszeit sehr viel mehr beachtet, als sich beispielsweise nach der Lektüre der Lehrbücher aus dieser Zeit vermuten liesse ${ }^{26}$. Diese Beobachtung gilt in der Nachkriegszeit für beide Teile Deutschlands. Die meisten dieser Veröffentlichungen lassen sich Themenfeldern zuordnen, die bereits während des 1 . Weltkriegs und in der Zwischenkriegszeit oder sogar schon um 1900 aktuell waren. Die Beiträge beschäftigen sich überwiegend mit den Auswirkungen des Krieges und der Kriegsgefangenschaft und - als wichtigstes neues Themenfeld nach dem 2. Weltkrieg - mit den Folgen der KZ-Haft.

Das Kriegsende 1945 markiert eine deutliche Zäsur im Umgang mit dem Thema «psychisches Trauma». Nur noch sehr vereinzelt erschienen Artikel, die auf prädisponierende Persönlichkeitsstrukturen oder Psychopathie abhoben ${ }^{27}$. Als erster Beitrag in der ersten Nachkriegsausgabe des Nervenarzt im Jahr 1947 steht Karl Bonhoeffers Resümee der Kriegserfahrungen an prominenter Stelle. Mit seinem Aufsatz «Vergleichende psychopathologische Erfahrungen aus den beiden Weltkriegen» aktualisierte Bonhoeffer gewissermassen den fünfundzwanzig Jahre zuvor veröffentlichten Beitrag zum 1. Weltkrieg ${ }^{28}$. Bei einer vergleichenden Betrachtung der beiden Texte fällt ein sehr viel vorsichtigerer sprachlicher Duktus im Jahre 1947 auf. Bonhoeffers Nachdenklichkeit und Zweifel wurden wahrscheinlich durch seine persönliche Betroffenheit durch die KZ-Haft, Folter und Hinrichtung seiner

24 Vgl. Hoffmann 1929; Hoffmann 1930; Payk 1972.

25 Bonhoeffer 1922, 44.

26 Kloocke et al. 2005.

27 Müller-Suur 1954; Becker 1959; Mayer 1960.

28 Bonhoeffer 1922; Bonhoeffer 1947. 
Söhne und Schwiegersöhne mitgeprägt. In diesem Text führte Bonhoeffer den für die Diskussion in der deutschen Nachkriegspsychiatrie wichtigen Ausdruck «Grenze der psychischen Tragfähigkeit» ein und formuliert als Forschungsdesiderat eine wissenschaftliche Untersuchung von KZ-Opfern, die Folterungen durchgemacht hatten. In Anbetracht von Bonhoeffers Autorität in der Traumadebatte seit mehr als 30 Jahren erstaunt es nicht, dass dessen persönlicher Meinungswandel einen weitreichenden Nachhall in der Nachkriegsentwicklung haben sollte. Das Modell der «Grenze der psychischen Tragfähigkeit» wurde von Bonhoeffer übrigens weiterhin, zumindest teilweise, als organische Schädigung konzeptionalisiert. Bonhoeffer sprach von einem «Zusammenwirken exzessiven affektiven Erlebens und körperlicher Qual» und benannte als physische Faktoren das Fehlen von Nahrung und Ausruhmöglichkeit sowie Fesselung, Stösse von Eisenstangen und Gewehrkolben gegen den Hinterkopf und Schläge auf die gefesselten Hände ${ }^{29}$.

Bonhoeffer sprach von der «Grenze der psychischen Tragfähigkeit» ursprünglich im Kontext der reaktiven Psychosen. Untersuchungen an Kriegsgefangenen waren in den ersten Nachkriegsjahren das Hauptanwendungsfeld für diese Diskussion ${ }^{30}$. Rommelsbacher apostrophierte Kriegsgefangenenlager sogar als «hochbedeutsames massenpsychologisches Experiment $»^{31}$. Fast alle diese Autoren nahmen zustimmend Bezug auf Bonhoeffers Lehre von der überschrittenen seelischen Toleranzgrenze bei zusätzlichem Einwirken von organischen Faktoren. Die so charakterisierten Psychosen wurden weiter als exogene Psychosen oder symptomatische Psychosen bezeichnet.

Vor dem Hintergrund einer von der Mehrzahl der Nachkriegspsychiater als organisch konzeptualisierten exogenen Psychose rückte auch das Bild der Dystrophie, das besonders bei heimkehrenden Kriegsgefangenen aus Russland beobachtet wurde, in das Interesse der Diskussion ${ }^{32}$. Unter Dystrophie wurde ein Mischbild aus extremer Mangelernährung und schwerer, oft durch Infektionskrankheiten bedingter physischer Erschöpfung verstanden, bei dem häufig auch psychische Veränderungen beobachtet wurden. Etliche Nachkriegsarbeiten befassten sich auch mit den Begriffen «Erschöpfung ${ }^{33}$ und «seelische Überforderung $»^{34}$.

29 Bonhoeffer 1947, 3.

30 Kröber 1948; Gerstacker 1949; Lewin 1949; Rommelspacher 1949; Gottschick 1950a; Gottschick 1950b; Schmitz 1951; Schilf 1960.

31 Rommelspacher 1949, 284.

32 Faust 1952; Herberg/Schilf 1958; Soyka 1958; Schilf 1960.

33 Rost 1949; Barylla 1952; Lippert 1959; Oles 1959; Schultz 1961.

34 Wegener 1953; Wieser 1962; Wieser et al. 1962; Wieser 1964. 
Ähnlich wie Kleist mehr als drei Dekaden zuvor, distanzierten sich Gottschick und Sneshewski von Bonhoeffers Position und sprachen von rein psychogenen Psychosen ${ }^{35}$. Gottschick berichtete eine Zunahme der Inzidenz von Psychosen bei deutschen Kriegsgefangenen in den USA, die nach seiner Ansicht fälschlich als Schizophrenien eingeordnet würden, während er einen direkten, nicht organisch vermittelten Kausalzusammenhang zwischen Gefangenschaft und Psychose sah. Diese Position blieb jedoch nicht unwidersprochen $^{36}$. Und wie schon seit den 1920er Jahren diskutieren etliche Beiträge die Frage der Psychogenese der Schizophrenien oder affektiven Störungen durch Traumen ${ }^{37}$. Getrennt von diesen Themenfeldern sind Beiträge zu traumatischen oder posttraumatischen Psychosen zu sehen, die ausschliesslich organische Psychosen nach einem physischen Trauma, meist einem SchädelHirn-Trauma, behandelten ${ }^{38}$.

Für das Thema KZ-Haft und Folgen der Verfolgung, das Bonhoeffer 1947 erstmalig aufgeworfen hatte, bot fast ausschliesslich der westdeutsche Nervenarzt ein Diskussionsforum. Zwischen 1954 und 1964 erschienen im Nervenarzt dreizehn Beiträge zu diesem Thema. Besonders in den ersten Jahren wurden KZ-Schäden häufig als organisch überlagert geschildert. Die Autoren sprachen von vegetativer, vegetativ-hormonaler oder vaso-vegetativer Dystonie oder von der vegetativ-emotionalen Alteration. Die Terminologie entwickelte sich schrittweise vom unspezifischen Verfolgungsschaden über erlebnisbedingten Verfolgungsschaden und erlebnisbedingten Spätschaden zum erlebnisreaktiven Persönlichkeitswandel nach Extrembelastung. Dieser Begriffswandel steht für einen fundamentalen Wandel im Krankheitskonzept, in dem sowohl (a) das auslösende Trauma (extrem), (b) der zeitliche Rahmen (spät einsetzend, andauernd) als auch (c) die Art der Psychopathologie (Wandel der Persönlichkeit statt neurotische Beschwerden) neu konzeptualisiert wurden. Diese Debatte sprengte den etablierten Rahmen der westdeutschen akademischen Psychiatrie. Sie verlief international und äusserst kontrovers ${ }^{39}$. Einige Diskussionsbeiträge dieser Teildebatte nahmen Bezug auf psychoanalytische Autoren und Konzepte, was bis in die jüngere Gegenwart eine Ausnahme innerhalb der deutschen psychiatrischen Traumadiskussion darstellt und auf den Einfluss der amerikanischen psychiatri-

35 Gottschick 1950a; Gottschick 1950b; Sneshewski 1955.

36 Schmitz 1951.

37 Elsässer 1957; Huszag 1958; Pauleikhoff 1958/59; Petrilowitsch 1959; Uschakov 1959; Blankenburg 1964.

38 Bay/Siebert 1951; Meyer 1954; Suckow 1954; Brendel 1955; Hubach 1960; Kisker 1960; Hentel 1964.

39 Strauss 1957; Bensheim 1960; Trautmann 1961; Levinger 1962; Witter 1962; von Baeyer et al. 1963. 
schen Tradition hinweist. Beispielsweise Edgar Trautmann aus New York zitierte Franz Alexander, Karen Horney, Abraham Kardiner und H. Spiegel ${ }^{40}$. Exponierte Vertreter der Debatte wie z.B. von Baeyer ordneten sich selbst einer anthropologischen Tradition in der Psychiatrie zu. In Hinblick auf den Ost-West-Vergleich bildet die KZ-Opfer-Debatte ebenfalls eine Ausnahme: Während sonst in der Traumadebatte in Auswahl und Gewichtung der Themen und im regelhaften Bezug auf die gemeinsame Vorkriegstradition die theoretischen Unterschiede zwischen ost- und westdeutscher Psychiatrie eher gering erscheinen, beschränkte sich die KZ-Opfer-Debatte dieser Zeitperiode ausschliesslich auf die westdeutsche Psychiatrie.

$\mathrm{Zu}$ den nicht-psychotischen Störungen nach Trauma erschienen zwischen 1938 und 1964 in den von uns untersuchten Zeitschriften rund vierzig Beiträge. Das mag wenig erscheinen, insbesondere wenn man berücksichtigt, dass sich ein Gutteil davon mit Fragen der Rentenbegutachtung befasste. In der Terminologie ist auffällig, dass noch in den späten 1940er Jahren überwiegend die Termini «psychogene Reaktion» und «abnorme Erlebnisreaktion» verwendet wurden, während nach 1950 die meisten Autoren zu dem Neurose-Begriff zurückkehrten ${ }^{41}$.

\section{d) $1964-1989$}

In diesem Zeitabschnitt fanden wir lediglich 54 Beträge. Zwanzig von diesen fallen unter den Themenkomplex «Trauma und Psychose», die übrigen Beiträge sind weniger einheitlich in ihrer Themenwahl. Eine zunehmend weite Definition von Trauma in Richtung Erlebnis, exogene Schäden, psychoreaktiver Faktor, lebensgeschichtlicher Faktor oder Belastung durch Migration lässt sich in den späten sechziger und siebziger Jahren beobachten und deutet den fliessenden Übergang zu angrenzenden Forschungsgebieten wie der Life-event-Forschung oder der transkulturellen Psychiatrie an.

Die Einführung der Diagnose Post-Traumatic Stress Disorder (PTSD) in das amerikanische DSM-III im Jahre 1980 führte keineswegs zu einer raschen Veränderung der diagnostischen Gewohnheiten in den beiden deutschen Psychiatrien ${ }^{42}$. In der westdeutschen Psychiatrie der 1980er Jahren fanden sich theoretische Überlegungen zu traumatischen Ereignissen wiederholt in

40 Trautmann 1961.

41 Krauss 1947; von Baeyer 1948; Henßge 1949; Krenz 1949; Rommelspacher 1949.

42 Kloocke et al. 2005. 
Beiträgen zur Life-Event-Forschung ${ }^{43}$. Fragen der Quantifizierung von traumatischen Ereignissen, die so genannte «Kausalattribution» zwischen Ereignis und Symptomen, die biologische Vermittlung von Stressreaktionen, aber auch Fragen der Prävention von Belastungsreaktionen wurden in diesem Kontext diskutiert. Etliche Untersuchungen befassten sich mit den Rentenneurosen, ohne jedoch das PTSD-Konzept explizit zu erwähnen ${ }^{44}$. In der DDR-Psychiatrie der achtziger Jahre fanden wir keinen Beitrag, der die neue Diagnose erwähnte.

Das bis 1970 sehr wichtige Thema «Trauma und Psychose» trat mehr und mehr in den Hintergrund. Hoffmann et al. veröffentlichten 1982 eine kasuistische Arbeit über das Ganser-Syndrom. Eine fortgesetzte Debatte zu den psychogenen Psychosen in der skandinavischen Tradition führten Degkwitz 1985, Strömgren 1986, Windgassen 1986 und Degkwitz 1987.

Die kontroverse Diskussion zu KZ-Opfern wurde in Westdeutschland zwischen 1964 und 1970 mit sieben Beiträgen fortgesetzt ${ }^{45}$. Und 1966 fand die psychische Traumatisierung von KZ-Opfern erstmals Erwähnung in der ostdeutschen Zeitschrift Psychiatrie, Neurologie und medizinische Psychologie. Der für KZ-Opfer engagierte Leipziger Psychiater Dietfried Müller-Hegemann, selbst ein Betroffener, verfasste ab 1966 drei Buchbesprechungen zu Publikationen aus dem westlichen Ausland ${ }^{46}$. Danach brach die Beschäftigung mit dem Thema «KZ-Opfer» ab. Erst 1989, nach einer Pause von 19 Jahren, veröffentlichte Peters in den Fortschritten einen wichtigen historischen Überblick über das Überlebenden-Syndrom.

\section{e) $1990-2005$}

Seit 1990 konnten wir 49 relevante Beiträge zu psychischer Traumatisierung identifizieren, mit einem klaren Anstieg des Interesses in den letzten Jahren und allein acht Beiträgen im Jahr 2005.

Ein Beitrag von Dreßing und Berger im Nervenarzt aus dem Jahr 1991 thematisierte die posttraumatische Stresserkrankung erstmals explizit ${ }^{47}$. Weitere Beiträge zur posttraumatischen Belastungsstörung folgten 1996 und 1997,

43 Cooper 1980; Katschnik 1980; Siegrist 1980; Matussek/May 1980/81; Malzacher et al. 1981; Möller et al. 1984/85.

44 Strasser 1974; Mascher 1976; Teusch/Engelmeier 1982; Teusch 1984; Foerster 1987.

45 Henseler 1965; Kluge 1965; Hoppe 1966; Henseler 1967; Luthe 1968; Venzlaff 1969; von Baeyer 1970.

46 Müller-Hegemann 1966a; Müller-Hegemann 1966b; Müller-Hegemann 1969.

47 Dreßing/Berger 1991. 
von da an wurde im Nervenarzt und den Fortschritten regelmässig zum Thema publiziert ${ }^{48}$. Inhaltlich auffallend ist, dass sich mehrere dieser Beiträge mit der Grenzbestimmung des Krankheitskonzeptes beschäftigten und beispielsweise Sack beklagte, das Spektrum der Symptome, die durch Traumatisierungen ausgelöst werden könnten, sei «weitaus umfangreicher, als es in den eng umgrenzten diagnostischen Kategorien der PTBS erfasst wird $»^{49}$.

Andere Beiträge zur Traumadebatte sind heterogener in ihrer Themenwahl: Ein umfangreicher Übersichtsartikel Jatzko et al. stellt internationale Ergebnisse zu bildgebenden Verfahren bei der PTSD dar ${ }^{50}$. In gewisser Hinsicht eine Umkehrung der häufig postulierten Kausalrelation von Trauma und Psychose zieht Hohl-Radke mit dem Konzept der psychoseinduzierten posttraumatischen Belastungsstörung in Betrach $t^{51}$. Mehrere Autoren schreiben über Syndrome im Grenzgebiet zu organischen Traumen und Erkrankungen $^{52}$. Weitere Arbeiten beschäftigen sich mit Klassifikationsfragen innerhalb des psychiatrischen Spektrums. Klosterkötter hinterfragt die Wirkungsweise von Traumata per $\mathrm{se}^{53}$. Zwei Beiträge nähern sich dem Thema «Trauma» konzeptionell auch aus psychoanalytischer Perspektive ${ }^{54}$. Andere Autoren behandeln praxisorientiert Fragen der Therapie und Prävention ${ }^{55}$ und der Begutachtung ${ }^{56}$ von psychischen Traumen.

Seit den 90er Jahren erschienen auch wieder einige Veröffentlichungen zu psychischer Traumatisierung in der NS-Zeit ${ }^{57}$ und zu den Opfern politischer Gewalt in der DDR ${ }^{58}$. Die Veröffentlichungen zu KZ-Opfern heben sich durch die Art ihrer Fragestellung von früheren Arbeiten ab: Bunk und Bronisch untersuchen sehr spezifische Aspekte der KZ-Haft, wie zum Beispiel die Beziehungsdynamik bei traumatisierten Kindern oder die Suizidalität. Tölles Arbeit hingegen nimmt, im Gegensatz zu der zumeist untersuchten Extremsituation im KZ, das Verfolgungstrauma im Alltag in den Blick.

48 Ebbinghaus et al. 1996; Bronisch 1997.

49 Sack 2004, 451.

50 Jatzko et al. 2005.

51 Hohl-Radke 2005.

52 Podoll et al. 2000; Leweke et al. 2001; Krauseneck et al. 2005.

53 Cima et al. 2003; Linden et al. 2004; Sack 2004; Giesbrecht/Merckelbach 2005; Hohl-Radke 2005; Klosterkötter 2005.

54 Frommer 2002; Wöller 2005.

55 Ebbinghaus et al. 1996; Schützwohl 2000.

56 Weber et al. 1998; Grobe 1999.

57 Bunk/Eggers 1993; Bronisch 1995; Ody 1998; Tölle 1999.

58 Peters 1991; Priebe et al. 1993; Priebe et al. 1994; Denis et al. 1997; Frommer 2002. 


\section{Diskussion}

Die Debatte um psychisches Trauma kann in der deutschen Psychiatrie auf eine lange Tradition zurückgreifen. Die Diskussion wurde bis Ende der 1960er Jahre sehr intensiv geführt und ebbte danach ab. Erst in jüngerer Zeit finden sich wieder mehr Veröffentlichungen zum Thema. Ein Schwerpunkt der historischen Debatte galt der Frage nach dem Zusammenhang von Trauma und Psychose. Wir konnten zeigen, dass in der deutschen Tradition bis in die Gegenwart häufig organische Interpretationen herangezogen wurden, um den Zusammenhang von Trauma und psychischen Symptomen zu verstehen.

Der Versuch, einen Überblick über die Fachdiskussion zu psychischer Traumatisierung in der deutschen Psychiatrie über einen Zeitraum von 117 Jahren zu geben, hat notwendigerweise Ausschnittcharakter. Die Fokussierung der Untersuchung auf psychiatrische Fachzeitschriften bedeutet eine erhebliche Einschränkung, da wir damit wichtige Facetten des Themas ausklammern, wie z.B. die Sichtweisen der Betroffenen oder der Öffentlichkeit und den Einfluss von gesellschaftlichen Prozessen, staatlichen Rahmenbedingungen, Gesetzesgrundlagen und konkreter Rechtsprechung auf die Entwicklung der Traumakonzepte. Zudem ist unsere Untersuchung auf die Psychiatrie Deutschlands beschränkt.

Die systematische Beschränkung unserer Quellenbasis auf bestimmte Zeitschriften führte dazu, dass einzelne wichtige Publikationen oder Konzepte (z.B. die Kraepelin'sche Schreckneurose) nicht oder nur kursorisch erwähnt werden. Ebenfalls fast völlig unberücksichtigt bleiben Beiträge von einem psychoanalytischen Hintergrund, da diese nicht in den klassischen psychiatrischen Publikationsorten veröffentlicht wurden. Andererseits bietet dieses strukturierte Vorgehen mit einer definierten Quellenbasis den Vorteil, dass auch weniger prominente Autoren zu Wort kommen. Somit entsteht ein Bild der Diskussion, das auch die theoretische «Wirksamkeit» eines Konzeptes, d.h. das Mass in dem es aufgegriffen, zitiert, kommentiert, modifiziert oder umgedeutet wurde, zur Abbildung bringt.

Unsere Untersuchung identifizierte rund 550 relevante Beiträge zu psychischer Traumatisierung, von denen der grösste Teil bis Ende der 1960er Jahre publiziert wurde. Typische Ereignisse, die im Laufe der Zeit als psychische Traumen diskutiert wurden, waren Unfälle, Hafterfahrungen, Dienst im Heer, Kriegserfahrungen von Heeresangehörigen und Zivilisten, Kriegsgefangenschaft, KZ-Haft und andere Formen der politischen Verfolgung. Nur sehr selten oder gar nicht wurde in unseren Quellen Bezug auf Natur- 
katastrophen, technische Katastrophen ${ }^{59}$ oder sexuelle Gewalt genommen. Die wichtigsten von uns identifizierten Themenkomplexe waren die Frage nach dem Zusammenhang von Trauma und Psychose, die Frage nach «neurotischen» oder «reaktiven» Störungen und die Frage nach den Auswirkungen der KZ-Haft.

Die Beschreibungen der Symptome und Syndrome, die dem Einwirken von traumatischen Ereignissen zugeschrieben wurden, waren äusserst vielfältig und decken das gesamte Spektrum der psychiatrischen Diagnostik ab. Immer wieder beklagten psychiatrische Experten, wie unspezifisch die geschilderten Symptome seien und wie schwierig es sei, klare Krankheitsbilder im Zusammenhang mit traumatischen Ereignissen abzugrenzen. Ähnlich vielfältig und wenig klar abgegrenzt war die psychiatrische Terminologie, die in der Diskussion verwendet wurde. Im Kontrast zu diesem Wildwuchs an Begriffen konnten wir für den Untersuchungszeitraum fünf dominierende Krankheitsmodelle etablieren:

1.Psychoreaktive Symptome nach traumatischen Ereignissen wurden spätestens ab 1916 als «hysterische» oder «abnorme Reaktionen» infolge einer psychopathischen oder degenerativen Anlage eingeordnet. Eine Entschädigung dieser Patienten, insbesondere von Kriegsteilnehmern, wurde grundsätzlich abgelehnt.

2. Psychosen im Rahmen der Haft wurden ebenfalls zumeist dem hysterischen Spektrum zugeordnet und eine degenerative Anlage postuliert. Alle Autoren betonen die gute Prognose und häufig auch den Aspekt des sekundären Krankheitsgewinns und der Simulation.

3. Für eine weitere Gruppe von Psychosen nach traumatischen Erlebnissen postulierte Bonhoeffer einen organisch vermittelten Pathomechanismus (z.B. Mangelernährung, Infektionen). Er nannte diese Präsentationen «exogene Reaktionstypen».

4. Unter dem Einfluss der Erfahrungen im 2. Weltkrieg entwickelte Bonhoeffer 1947 das Konzept der «Grenze der seelischen Belastbarkeit». Aus diesem Ansatz ging in mehreren Schritten das Modell des «erlebnisbedingten Persönlichkeitswandels» bei KZ-Folteropfern hervor, das per definitionem auf schwerste traumatische Erfahrungen beschränkt blieb. Dieses Krankheitsmodell wurde die Grundlage für die Anerkennung und Entschädigung von anhaltenden psychischen Spätschäden bei KZ-Opfern.

5. Das amerikanische PTSD-Konzept wurde in unseren Quellen mit einer deutlichen Verzögerung rezipiert und erst ab circa 2000 regelhaft als Referenzmodell für psychische Traumatisierung herangezogen.

59 Mende/Ploeger 1966; Ploeger 1969; von der Stein et al. 1992. 
Unsere Darstellung des Ablaufs der historischen Diskussion basiert auf einigen theoretischen Implikationen: Bereits für die Auswahl der zu analysierenden Beiträge war die Frage zentral, welche Art von Ereignis in der jeweiligen Zeit als psychisches Trauma angesehen wurde. Der Begriff «psychisches Trauma» umfasst ein weites Spektrum von Vorstellungsinhalten und Krankheitsmodellen. Wodurch jedoch ein belastendes Ereignis letztlich als psychisches Trauma wahrgenommen wird, hängt weniger von Eigenschaften des Ereignisses oder von fachlichen Kriterien, als von ausserhalb der Medizin liegenden Faktoren $a^{6}{ }^{60}$. Pross konnte zeigen, dass die Anerkennung der KZ-Folgen als psychisches Trauma trotz Bonhoeffers Überlegungen zu den Grenzen der psychischen Tragfähigkeit keineswegs von Psychiatern entwickelt wurde, sondern nur unter massivem politischem Druck in die Psychiatrie hineingetragen wurde ${ }^{61}$. Ähnliches gilt auch für die Konzepte der Unfallneurosen, der Haftpsychosen, der Kriegsneurosen, aber auch der PTSD, die durch die Wirksamkeit von Interessengruppen von aussen Eingang in die Psychiatrie fanden. Besonders das Beispiel der Haftpsychosen zeigt, wie juristische Fragestellungen (die Frage nach der Schuld- bzw. Haftfähigkeit) und individuelle Interessen (z.B. der Wunsch nach Haftverschonung) auf sehr spezifische Weise auf die medizinische Debatte einwirkten, indem sie die Simulationserkennung zu einer zentralen Frage machten. Die aus dieser Konstellation resultierende «Kriminalisierung» der traumatischen Psychosen und die nachfolgende Verknüpfung mit der Degenerationsdebatte befestigten eine bereits zuvor existierende ablehnende oder skeptische Haltung innerhalb der Psychiatrie. Die für Laien so plausible Vorstellung, dass psychische Erkrankungen oder Symptome durch äussere Ereignisse ausgelöst werden können, schien dem Erfahrungswissen psychiatrischer Experten diametral entgegenzustehen und nur unter Widerstand akzeptiert werden zu können. Zukünftige Studien, die weiter reichende Quellen ausserhalb der akademischen Psychiatrie einbeziehen sollten, müssten die Gründe für diese anhaltende Konfliktsituation untersuchen.

Esther Fischer-Homberger rekonstruierte detailliert, wie sich in der Diskussion um die traumatische Neurose das Trauma weg vom Organischen, hin zu einer psychologischen Interpretation entwickelte ${ }^{62}$. In unserem Quellenmaterial war diese Bewegung nicht, oder weniger deutlich, zu beobachten. Die Diskussion blieb bis in die jüngste Gegenwart sehr stark einem organischen Krankheitsmodell und sogar einem organischen Modell von Trauma verhaftet. Wir haben dieses Phänomen dahin gehend interpretiert, dass sich

60 Fischer-Homberger 1970.

61 Pross 1988.

62 Fischer-Homberger 1975, 54. 
die Entwicklung hin zu einer psychologischen Interpretation auf die engere Debatte zur traumatischen Neurose beschränkte, die aber innerhalb der akademischen Psychiatrie eine randständige Position einnahm. Andere parallel oder später existierende Traumakonzepte hingegen zeichneten sich nahezu ausnahmslos durch eine enge Verknüpfung mit organischen Faktoren aus. Es mag sein, dass eine zumindest teilweise organische Deutungsmöglichkeit Voraussetzung dafür war, dass sich ein Traumakonzept innerhalb der akademischen Psychiatrie überhaupt etablieren konnte. Bonhoeffer kam diesbezüglich eine zentrale Rolle zu. Er formulierte bereits 1911 unter Verweis auf Birnbaum eine ätiologische Dreiteilung, welche psychische Folgen von Traumen entweder als (a) (Re-)Aktivierung einer latent vorhandenen endogenen Erkrankung, (b) als organisch mitbedingt oder (c) auf dem Boden einer minderwertigen Konstitution entstanden einordnete ${ }^{63}$. Diese ätiologische Dreiteilung mit ihrer nachhaltig ordnenden Wirkung innerhalb eines naturwissenschaftlichen Denkmodells wurde Konsens und dominierte die Fachdiskussion in den kommenden fünf Jahrzehnten und darüber hinaus.

\section{Schlussfolgerung}

Das Thema «psychisches Trauma» wurde in Deutschland seit dem ausgehenden 19. Jahrhundert intensiv diskutiert. Dem für Laien - und auch für die Psychiater des frühen 19. Jahrhunderts - unmittelbar plausiblen Zusammenhang zwischen erschütterndem Erlebnis und psychischen Symptomen stand eine erhebliche Skepsis der psychiatrischen Experten gegenüber. Eine mögliche Erklärung für diese Konstellation ist nach unserer Einschätzung die Entwicklung des naturwissenschaftlichen Krankheitsmodells in der Psychiatrie. Die Einbindung des psychischen Traumas in das naturwissenschaftliche Modell erwies sich als schwierig und widersprüchlich. Traumakonzepte konnten sich in der Folge nur dann in der akademischen Psychiatrie etablieren, wenn sie - zumindest teilweise - organisch deutbar waren.

\section{Bibliographie}

Berger, Georg, Die beratenden Psychiater des deutschen Heeres 1939-1945 (Frankfurt a. M. 1998) Blaßneck, Klaus, Militärpsychiatrie im Nationalsozialismus: Kriegsneurotiker im Zweiten Weltkrieg (Würzburg 2000)

Bonhoeffer, Karl, «Zur Frage der Klassifikation der symptomatischen Psychosen», Berliner Klinische Wochenschrift 45 (1908) 2257-2260

63 Bonhoeffer 1911. 
- Die symptomatischen Psychosen im Gefolge von akuten Infektionen und inneren Erkrankungen (Leipzig/Wien 1910)

- «Wie weit kommen psychogene Krankheitszustände und Krankheitsprozesse vor, die nicht der Hysterie zuzurechnen sind?», Allgemeine Zeitschrift für Psychiatrie und psychischgerichtliche Medizin 68 (1911) 519

- «Über die Bedeutung der Kriegserfahrungen für die allgemeine Psychopathologie und Ätiologie der Geisteskrankheiten», in: Otto von Schjerning (Hrsg.), Handbuch der Ärztlichen Erfahrungen im Weltkriege 1914/1918, Bd. IV, Geistes- und Nervenkrankheiten (Leipzig 1922) 3-44

- «Beurteilung, Begutachtung und Rechtsprechung bei den sogenannten Unfallneurosen», Deutsche Medizinische Wochenschrift 52 (1926) 179-182

Eghigian, Greg, «Die Bürokratie und das Entstehen von Krankheit. Die Politik und die Rentenneurosen 1890-1926», in: Jürgen Reulecke/Adelheid Gräfin zu Castell Rüdenhausen (Hg.), Stadt und Gesundheit. Zum Wandel von «Volksgesundheit» und kommunaler Gesundheitspolitik im 19. und frühen 20. Jahrhundert (Stuttgart 1991)

Feilchenfeld, Leopold, Lehrbuch der praktischen Versicherungsmedizin (Berlin 1927)

Fischer-Homberger, Esther, «Der Begriff 〈Krankheit〉 als Funktion aussermedizinischer Gegebenheiten. Zur Geschichte der traumatischen Neurose», Sudhoffs Archiv 54 (1970) 225-241

- Die traumatische Neurose. Vom somatischen zum sozialen Leiden (Bern/Stuttgart/Wien 1975)

- «Zur Medizingeschichte des Traumas», Gesnerus 56 (1999) 260-294

Goltermann, Sonja, «Psychisches Leid und herrschende Lehre. Der Wissenschaftswandel in der westdeutschen Psychiatrie der Nachkriegszeit», in: Bernd Weisbrod (Hrsg.), Akademische Vergangenheitspolitik. Beiträge zur Wissenschaftskultur der Nachkriegszeit (Göttingen 2002) 243-280

Hilpert, Ronald, Rekonstruktion der Geschichte eines speziellen Elektrosuggestivverfahrens («Pansen») aus Archivmaterialen des Heeressanitätswesens der Wehrmacht und dessen Einordnung in das Kriegsneurosenproblem des Zweiten Weltkrieges (Leipzig 1995) Diss. med. dent.

Kloocke, Ruth/Heinz-Peter Schmiedebach/Stefan Priebe, «Psychisches Trauma in deutschsprachigen Lehrbüchern der Nachkriegszeit - die psychiatrische «Lehrmeinung» zwischen 1945 und 2002», Psychiatrische Praxis 32 (2005) 327-333

- «Trauma und Psychose in der deutschen Psychiatrie. Eine historische Analyse», Psychiatrische Praxis 36 (2010) 142-147

Komo, Günther, Für Volk und Vaterland. Die Militärpsychiatrie in den Weltkriegen (Münster/Hamburg 1992)

Lembach, Frank, Die «Kriegsneurose» in deutschsprachigen Fachzeitschriften der Neurologie und Psychiatrie von 1889 bis 1922 (Mannheim 1998) Diss. med.

Lerner, Paul, Hysterical Men. War, Psychiatry, and the Politics of Trauma in Germany, 1890-1930 (Ithaca/London 2003)

Micale, Mark S./Paul Lerner (eds), Traumatic Pasts. History, Psychiatry, and Trauma in the Modern Age, 1870-1930 (Cambridge/New York 2001)

Müller, Roland, Wege zum Ruhm: Militärpsychiatrie im Zweiten Weltkrieg - das Beispiel Marburg (Köln 2001)

Oppenheim, Hermann, Die traumatischen Neurosen nach den in der Nervenklinik der Charité in den letzten 5 Jahren gesammelten Beobachtungen (Berlin 1889)

Payk, Theo Rudolf, «Beitrag zur Frage der Auslösung einer akuten Schizophrenie durch psychische Erschütterung», Schweizer Archiv für Neurologie und Psychiatrie 111 (1972) 131-141

Pross, Christian, Wiedergutmachung. Der Kleinkrieg gegen die Opfer (Frankfurt am Main 1988)

Riedesser, Peter/Axel Verderber, «Maschinengewehre hinter der Front». Zur Geschichte der deutschen Militärpsychiatrie (Frankfurt a. M. 1996)

Roth, Karl-Heinz, «Die Modernisierung der Folter», 1999, Zeitschrift für Sozialgeschichte des 20. und 21. Jahrhunderts 2 (1987) 6-75

Stier, Ewald, Über die sogenannten Unfallneurosen (Leipzig 1926)

Thomann, Klaus-Dieter/Michael A. Rauschmann, «Die <posttraumatische Belastungsstörung>historische Aspekte einer 〈modernen〉 psychischen Erkrankung im deutschen Sprachraum», Medizinhistorisches Journal 28 (2003) 103-138 
Tölle, Rainer, «Die «Kriegsneurose» - ein frühes Modell der pluridimensional verstandenen psychiatrischen Traumatologie», Psychiatrische Praxis 32 (2005) 336-341

Wildgrube, Christiane/Sara Dimic/Ruth Kloocke/Heinz-Peter Schmiedebach/Stefan Priebe, «Psychologial trauma in German, Serbian and British psychiatry since 1945 - a comparison of textbooks», in: Waltraud Ernst/Thomas Mueller (eds), Transnational Psychiatry in Comparative Perspective, c. 1800-2000 (in print 2010)

\section{Quellen}

Abenheimer, Karl, «Zivilrechtliche Haftung für Unfallneurosen», Der Nervenarzt 6 (1933) $525-534$

Anonym, «Referat zu Philippi. Strafvollzug und Verbrecher», Archiv für Psychiatrie und Nervenkrankheiten 49 (1912) 1014

Anonym, «Referat zu Homburger A (1912) Lebensschicksale geisteskranker Strafgefangener», Archiv für Psychiatrie und Nervenkrankheiten 52 (1913) 848

Bähmig, «Hysterische Unfallerkrankungen bei Telephonistinnen», Archiv für Psychiatrie und Nervenkrankheiten 37 (1903) 1050f.

Baeyer, Walter von, «Zur Statistik und Form der abnormen Erlebnisreaktionen in der Gegenwart», Der Nervenarzt 19 (1948) 402-408

- «Psychiatrisches Gutachten über Fragen der Glaubwürdigkeit und Erinnerungszuverlässigkeit bei der Beurteilung von Zeugenaussagen rassisch Verfolgter, die weit zurückliegenden Extrembelastungen ausgesetzt waren», Der Nervenarzt 41 (1970) 83-89

- /Heinz Häfner/Karl Peter Kisker, «Wissenschaftliche Erkenntnis〉 oder <menschliche Wertung> der erlebnisreaktiven Schäden Verfolgter?», Der Nervenarzt 34 (1963) 120-123

Barylla, Fritz, «Psychopathogenese zeitbedingter Erschöpfungszustände», Psychiatrie, Neurologie und medizinische Psychologie 4 (1952) 63

Bay, Eberhard/Peter Siebert, «Zur Pathophysiologie der traumatischen Psychose», Der Nervenarzt 22 (1951) 52-55

Becker, A. M., «Zur Typengliederung der Psychopathie», Der Nervenarzt 30 (1959) 159-170

Bensheim, H., «Die K.Z.-Neurose rassisch Verfolgter», Der Nervenarzt 31 (1960) 462-469

Blankenburg, Wolfgang, «Lebensgeschichtliche Faktoren bei manischen Psychosen», Der Nervenarzt 35 (1964) 536-539

Bonhoeffer, Karl, «Die exogenen Reaktionstypen», Archiv für Psychiatrie und Nervenkrankheiten 58 (1917) 58-70

- «Vergleichende psychopathologische Erfahrungen aus den beiden Weltkriegen», Der Nervenarzt 18 (1947) 1-4

Brendel, F., «Zur Therapie der akuten posttraumatischen Psychosen», Der Nervenarzt 26 (1955) $293 f$.

Bresler, J., «Neurosen. Bericht über die psychiatrische Literatur im Jahre 1916», in: Otto Snell (Hrsg.), Bericht über die psychiatrische Literatur im Jahre 1916. Literaturheft zu Band 74, Allgemeine Zeitschrift für Psychiatrie und psychisch-gerichtliche Medizin (Berlin 1919) 62-96

Bronisch, Thomas, «Suizidalität unter Extrembelastung», Fortschritte der Neurologie, Psychiatrie 63 (1995) 139-148

- «Posttraumatic Stress Disorder - Posttraumatische Belastungsstörungen», Fortschritte der Neurologie, Psychiatrie 65 (1997) 195-207

Bumke, Otto/Rosenfeld, «Referat zu Homburger A, Lebensschicksale geisteskranker Strafgefangener», Archiv für Psychiatrie und Nervenkrankheiten 46 (1910) 811f.

Bunk, Detlef/Christian Eggers, «Die Bedeutung beziehungsdynamischer Faktoren für die Psychopathogenese von im Kindesalter Naziverfolgten», Fortschritte der Neurologie, Psychiatrie 61 (1993) 38-45

Cima, Maaike/Harald Merckelbach/Sabine Hollnack/Erhard Knauer, «Der Zusammenhang zwischen Trauma und Dissoziation: Eine kritische Betrachtung», Fortschritte der Neurologie, Psychiatrie 71 (2003) 600-608

Cooper, B., «Die Rolle von Lebensereignissen bei der Entstehung von psychischen Erkrankungen», Der Nervenarzt 51 (1980) 321-331 
Degwitz, Rudolf, «Die psychogenen Psychosen. Eine Übersicht über die klinischen Bilder, die Genese, Prognose und Therapie», Fortschritte der Neurologie, Psychiatrie 53 (1985) 22-28

- «Stellungnahme zu den Bemerkungen von K. Windgassen zur Arbeit von E. Strömgren «Psychogene Psychosen»», Der Nervenarzt 58 (1987) 392

Denis, Doris/Jana Eslam/Stefan Priebe, «Psychische Störungen nach politischer Inhaftierung in der Sowjetischen Besatzungszone und der ehemaligen DDR von 1945-1972», Fortschritte der Neurologie, Psychiatrie 65 (1997) 524-530

Dreßing, Harald/Mathias Berger, «Posttraumatische Stresserkrankungen. Zur Entwicklung des gegenwärtigen Krankheitskonzepts», Der Nervenarzt 62 (1991) 16-26

Ebbinghaus, Ruth/Michael Bauer/Stefan Priebe, «Behandlung der posttraumatischen Belastungsstörung», Fortschritte der Neurologie, Psychiatrie 64 (1996) 433-443

Edel, Max, «Ueber Unfallpsychosen. Referat, vorgetragen auf der Jahresversammlung des Vereins deutscher Irrenärzte, 23.4.1901», Allgemeine Zeitschrift für Psychiatrie und psychischgerichtliche Medizin 58 (1901) 734-736

Elsässer, Günter, «Grundsätzliches zur Frage der Psycho- und Endogenese der endogenen Psychosen», Der Nervenarzt 28 (1957) 533-535

Faust, Clemens, «Hirnatrophie nach Hungerdystrophie», Der Nervenarzt 23 (1952) 406-412

Foerster, Klaus, «Die sogenannte 〈Rentenneurose〉 - psychopathologisches Syndrom oder obsoleter Begriff?», Fortschritte der Neurologie, Psychiatrie 55 (1987) 249-260

Frommer, Jörg, «Psychische Störungen durch globale gesellschaftliche Veränderungen. Zur politischen Traumatisierung der Bevölkerung in den neuen Bundesländern», Fortschritte der Neurologie, Psychiatrie 70 (2002) 418-428

Gerstacker, Wilhelm, «Vorüberlegungen zur allgemeinen Psychologie und Pathopsychologie des Krieges», Archiv für Psychiatrie und Nervenkrankheiten vereinigt mit Zeitschrift für die gesamte Neurologie und Psychiatrie 182 (1949) 31-50

Giesbrecht,Timo/Harald Merckelbach, «Über die kausale Beziehung zwischen Dissoziation und Trauma. Ein kritischer Überblick», Der Nervenarzt 76 (2005) 20-27

Gottschick, Johann, «Neuropsychiatrische Erkrankungen bei deutschen Kriegsgefangenen in USA im Lichte statistischer Betrachtungen», Archiv für Psychiatrie und Nervenkrankheiten 185 (1950a) 491-510

- «Kriegsgefangenschaft und Psychosen», Der Nervenarzt 21 (1950b) 129-132

Grobe, Thomas, «Anmerkungen zu der Arbeit von M. M. Weber, I. A. Antonijevic und T. Bronisch: Die versorgungsrechtliche Beurteilung einer posttraumatischen Belastungsstörung [Der Nervenarzt 69, 1998, 811-814]», Der Nervenarzt 70 (1999) 671f.

Grossmann, Schulze, «Referat zu Binswanger O. Ueber die Pathogenese und klinische Stellung der Erschöpfungspsychosen», Archiv für Psychiatrie und Nervenkrankheiten 29 (1896) 978-983

Gruhle, Hans W., «Zum Aufsatz über «Zivilrechtliche Haftung für Unfallneurosen` von Dr. Karl Abenheimer. Und Erwiderung von K. Abenheimer», Der Nervenarzt 6 (1933) 634-636

Hasche-Klünder, «Ueber atypisch verlaufende Psychosen nach Unfall», Archiv für Psychiatrie und Nervenkrankheiten 44 (1908) 668-708

Heinicke, W., «Zur Klinik der Gefängnispsychosen», Archiv für Psychiatrie und Nervenkrankheiten 48 (1911) 1091-1097

Henseler, Heinz, «Zum gegenwärtigen Stand der Beurteilung erlebnisbedingter Spätschäden nach Verfolgung», Der Nervenarzt 36 (1965) 333-338

- «Zum gegenwärtigen Stand der Beurteilung erlebnisbedingter Spätschäden nach Verfolgung. Stellungnahme zu dem Diskussionsbeitrag von K. D. Hoppe [Der Nervenarzt 37, 1966, 124]», Der Nervenarzt 38 (1967) 119f.

Henßge, «Reaktive psychische Erkrankungen der Nachkriegszeit», Psychiatrie, Neurologie und medizinische Psychologie 1 (1949) 133-137

Hentel, H., «Heautoskopie bei traumatischer Psychose. Zugleich ein Beitrag zur Phänomenologie der Heautoskopie», Archiv für Psychiatrie und Nervenkrankheiten 206 (1964) 727-735

Herberg, H. J./E. Schilf, «Psychopathologisches Syndrom nach jahrelanger Haft und schwerer Fehl- und Mangelernährung bei Spätestheimkehrern», Der Nervenarzt 29 (1958) 85f.

Hess, Eduard, «Ueber hysterisches Irresein», Allgemeine Zeitschrift für Psychiatrie und psychisch-gerichtliche Medizin 60 (1903) 214f. 
Hoffmann, Hermann, «Reaktive Psychosen und Neurosen», Fortschritte der Neurologie und Psychiatrie und ihrer Grenzgebiete 1 (1929) 174-184

- «Reaktive Psychosen und Neurosen», Fortschritte der Neurologie und Psychiatrie und ihrer Grenzgebiete 2 (1930) 137-151

Hohl-Radke, Felix, «Die psychoseinduzierte posttraumatische Belastungsstörung», Der Nervenarzt 76 (2005) 879-882

Hoppe, Klaus D., «Zum gegenwärtigen Stand der Beurteilung erlebnisbedingter Spätschäden nach Verfolgung. Bemerkungen zur gleichnamigen Arbeit von H. Henseler [Der Nervenarzt 36, 1965, 8]», Der Nervenarzt 37 (1966) 124

Hubach, Heinrioh, «Traumatische Psychose bei einem 8jährigen Mädchen, zugleich ein Beitrag zum kindlichen Mutismus», Der Nervenarzt 31 (1960) 281f.

Huszag, I., «Über den heutigen Stand und die Probleme der Schizophrenie-Forschung», Archiv für Psychiatrie und Nervenkrankheiten 197 (1958) 32-44

Jatzko, A./A. Schmitt/A. Kordon/D. F. Braus, «Bildgebende Befunde bei der posttraumatischen Belastungsstörung (PTBS): Literaturübersicht», Fortschritte der Neurologie, Psychiatrie 73 (2005) 377-391

Jossmann, Paul, «Über die Rechtsbegriffe <äußerer Anlass〉 und <innerer Zusammenhang〉 für die medizinische Beurteilung der Rentenneurose», Der Nervenarzt 2 (1929) 385-393

- «Über einige Grundbegriffe der Rentenneurose. Beitrag zur Aussprache in dieser Zeitschrift», Der Nervenarzt 3 (1930) 68-77

Katschnik, Heinz, «Methodische Probleme der Life-Event-Forschung», Der Nervenarzt 51 (1980) 332-343

Kisker, Karl Peter, «Zum Stellenwert konfabulatorischer Syndrome innerhalb akuter posttraumatischer Psychosen», Der Nervenarzt 31 (1960) 481-489

Kleist, Karl, «Schreckpsychosen», Allgemeine Zeitschrift für Psychiatrie und psychisch-gerichtliche Medizin 74 (1918a) 171f.

- «Schreckpsychosen», Allgemeine Zeitschrift für Psychiatrie und psychisch-gerichtliche Medizin 74 (1918b) 432-510

Klosterkötter, Joachim, «Was bewirkt das Trauma bei der posttraumatischen Belastungsstörung?», Fortschritte der Neurologie, Psychiatrie 73 (2005) 375f.

Kluge, Erich, «Über Ergebnisse bei der Begutachtung Verfolgter», Der Nervenarzt 36 (1965) 321

Krauseneck, T./H.-B. Rothenhäusler/G. Schelling/H.-P. Kapfhammer, «Posttraumatische Belastungsstörungen bei somatischen Erkrankungen», Fortschritte der Neurologie, Psychiatrie 73 (2005) 206-217

Krauss, Paul, «Zur Krampfbehandlung der Verstimmungszustände und psychogenen Reaktionen», Der Nervenarzt 18 (1947) 127-134

Krenz, H., «Zeitbedingte abnorme Erlebnisreaktionen», Allgemeine Zeitschrift für Psychiatrie und psychisch-gerichtliche Medizin 124 (1949) 336-357

Kröber, Ernst, «Über Haftpsychosen (Anlage- und exogene Faktoren bei der Entstehung von Haftreaktionen. Erfahrungen an Internierten.)», Der Nervenarzt 19 (1948) 408-413

Laehr, H., «Neurosen. Bericht über die psychiatrische Literatur im Jahre 1917», in: Otto Snell (Hrsg.), Bericht über die psychiatrische Literatur im Jahre 1917. II. Literaturheft zu Band 75, Allgemeine Zeitschrift für Psychiatrie und psychisch-gerichtliche Medizin (Berlin 1919) 173-210

Laquer, L./Buchholz, «14. Wanderversammlung der Südwestdeutschen Neurologen und Irrenärzte», Archiv für Psychiatrie und Nervenkrankheiten 21 (1890) 654-657

Levinger, L., «Psychiatrische Untersuchungen in Israel an 800 Fällen mit GesundheitsschädenForderungen wegen Nazi-Verfolgung», Der Nervenarzt 33 (1962) 75-80

Leweke, Frank, et al., «Ein Patient mit Golfkriegssyndrom? Zur Diskussion eines unklaren Krankheitsbildes», Der Nervenarzt 72 (2001) 541-545

Lewin, Bruno, «Neurologisch-psychiatrische Untersuchungen und Beobachtungen an deutschen Kriegsgefangenen in Ägypten 1941-1947», Psychiatrie, Neurologie und medizinische Psychologie 1 (1949) 230-236

Linden, Michael/B. Schippan/K. Baumann/R. Spielberg, «Die posttraumatische Verbitterungsstörung (PTED). Abgrenzung einer spezifischen Form der Anpassungsstörung», Der Nervenarzt 75 (2004) 51-57 
Lippert, E., «Schizoforme Psychosen bei Erschöpfungszuständen», Psychiatrie, Neurologie und medizinische Psychologie 11 (1959) 117

Luthe, R., ««Erlebnisreaktiver Persönlichkeitswandel〉 als Begriff der Begutachtung im Entschädigungsrecht», Der Nervenarzt 39 (1968) 465-467

Malzacher, Max/Jörg Merz/Daniel Ebnöther, «Einschneidende Lebensereignisse im Vorfeld akuter schizophrener Episoden», Archiv für Psychiatrie und Nervenkrankheiten 230 (1981) 227-242

Mascher, W. L., «Die Rentenneurose. Versuch einer Bestandsaufnahme», Der Nervenarzt 47 (1976) 417-423

Matussek, Paul/Ulrike May, «Verlustereignisse in der Kindheit als prädisponierende Faktoren für neurotische und psychotische Depressionen», Archiv für Psychiatrie und Nervenkrankheiten 229 (1980/81) 189-204

Mayer, Klaus, «Die Bedeutung der Persönlichkeitsstruktur für die Entwicklung seelischer Fehlhaltungen nach einem Unfall», Der Nervenarzt 31 (1960) 211-216

Mende, Werner/A. Ploeger, «Das Verhalten und Erleben von Bergleuten in der Extrembelastung des Eingeschlossenseins», Der Nervenarzt 37 (1966) 209-219

Merzbacher,Ludwig, «Einige statistische Bemerkungen über Unfallneurosen», Allgemeine Zeitschrift für Psychiatrie und psychisch-gerichtliche Medizin 64 (1907) 168

Meyer, Hans-Hermann, «Zur Klinik und Therapie der akuten posttraumatischen Psychosen», Der Nervenarzt 25 (1954) 462-465

Möller, Hans-Jürgen/C. Grießhammer/H. Hacker, «Einschneidende Lebensereignisse im Vorfeld von akuten psychischen Erkrankungen», European Archives of Psychiatry and Neurological Sciences 234 (1984/85) 118-124

Moerchen, F., «Über die Entschädigungspflicht 〈seelisch-nervöser〉 Unfallfolgen», Der Nervenarzt 1 (1928) 419-422

Müller-Hegemann, Dietfried, «Buchbesprechung: Eitinger L. Concentration Camp Survivors in Norway and Israel», Psychiatrie, Neurologie und medizinische Psychologie 18 (1966a) 439

- «Buchbesprechung: Baeyer W; Häfner H; Kisker KP. Psychiatrie der Verfolgten», Psychiatrie, Neurologie und medizinische Psychologie 18 (1966b) 356f.

- «Buchbesprechung: Herberg, HJ. Die Beurteilung von Gesundheitsschäden nach Gefangenschaft und Verfolgung», Psychiatrie, Neurologie und medizinische Psychologie 21 (1969) 441f.

Müller-Suur, Hemmo, «Erlebnishintergrund und Persönlichkeitshaltung», Der Nervenarzt 25 (1954) 431-433

Ody, Reinhard, «Zur Entschädigung der zwangssterilisierten Opfer des Nationalsozialismus. Ein noch immer nicht abgeschlossenes Kapitel», Der Nervenarzt 69 (1998) 815-817

Oles, M., «Schizoforme Psychosen bei Erschöpfungszuständen», Psychiatrie, Neurologie und medizinische Psychologie 11 (1959) 112-116

Pauleikhoff, Berhard, «Über die Auslösung endogener depressiver Phasen durch situative Einflüsse», Archiv für Psychiatrie und Nervenkrankheiten vereinigt mit Zeitschrift für die gesamte Neurologie und Psychiatrie 198 (1958/59) 456-470

Peters, Uwe Hendrik, «Die psychischen Folgen der Verfolgung. Das Überlebenden-Syndrom», Fortschritte der Neurologie, Psychiatrie 57 (1989) 169-191

- «Über das Stasi-Verfolgten-Syndrom», Fortschritte der Neurologie, Psychiatrie 59 (1991) 251-265

Petrilowitsch, Nikolaus, «Beitrag zur Frage der Provokation endogener Psychosen durch exogene Schäden», Archiv für Psychiatrie und Nervenkrankheiten vereinigt mit Zeitschrift für die gesamte Neurologie und Psychiatrie 198 (1959) 399-404

Ploeger, A., «Gruppendynamik in einer Extremsituation. Weitere Untersuchungen an den Überlebenden der Bergwerkskatastrophe von Legede 1963», Der Nervenarzt 40 (1969) 308-314

Podoll, Klaus/H. J. Kunert/Henning Saß, «Posttraumatische Belastungsstörungen bei neurogener Amnesie für das traumatische Ereignis», Fortschritte der Neurologie, Psychiatrie 68 (2000) 458-467

Priebe, Stefan/H. Rudolf/Michael Bauer/M. Häring, «Psychische Störungen nach politischer Inhaftierung in der DDR - Sichtweisen der Betroffenen», Fortschritte der Neurologie, Psychiatrie 61 (1993) 55-61

- /K. Bolze/K.H. Rudolf, «Andauernde psychische Störungen nach Repressalien infolge eines Ausreiseantrages in der damaligen DDR», Fortschritte der Neurologie, Psychiatrie 62 (1994) 433-437 
Raecke, Julius, «Ueber Erschöpfungspsychosen», Allgemeine Zeitschrift für Psychiatrie und psychisch-gerichtliche Medizin 57 (1900) 39-48

- «Hysterischer Stupor bei Strafgefangenen», Allgemeine Zeitschrift für Psychiatrie und psychisch-gerichtliche Medizin 58 (1901) 409-446

- «Zur Lehre vom hysterischen Irresein», Archiv für Psychiatrie und Nervenkrankheiten 40 (1905) 171-211

Richter, A., «Zwei Gutachten über traumatische Neurosen mit Irrsinn», Archiv für Psychiatrie und Nervenkrankheiten 22 (1891) 481-506

Riese, Walther, «Einige erkenntniskritische Bemerkungen zu den Ausführungen von Jossmann: Über die Bedeutung der Rechtsbegriffe 〈äußerer Anlaß〉 und <innerer Zusammenhang für die medizinische Beurteilung der Rentenneurose», Der Nervenarzt 3 (1930) 65-68

Rommelspacher, Franz, «Der Einfluß der Kriegsgefangenschaft auf Pathogenese und Pathoplastik von Psychosen und Erlebnisreaktionen», Archiv für Psychiatrie und Nervenkrankheiten vereinigt mit Zeitschrift für die gesamte Neurologie und Psychiatrie 182 (1949) 284-300

Rost, Joachim, «Beitrag zum psychischen Zustandsbild der Erschöpfung», Der Nervenarzt 20 (1949) $325 f$.

Rüdin, Ernst, «Ueber die klinischen Formen der Gefängnispsychosen», Allgemeine Zeitschrift für Psychiatrie und psychisch-gerichtliche Medizin 58 (1901) 447-462

- «Eine Form akuten halluzinatorischen Verfolgungswahns in der Haft ohne spätere Weiterbildung des Wahns und ohne Korrektur», Allgemeine Zeitschrift für Psychiatrie und psychisch-gerichtliche Medizin 60 (1903) 852-888

Sack, Martin, «Diagnostische und klinische Aspekte der komplexen posttraumatischen Belastungsstörung», Der Nervenarzt 75 (2004) 451-459

Schilf, Erich, «Über eine symptomatische Psychose mit schizophrener Symptomatik nach 3jähriger russischer Gefangenschaft mit Dystrophie», Der Nervenarzt 31 (1960) 176-178

Schmitz, Willi P., «Bemerkungen zu dem Aufsatz von J. Gottschick: Kriegsgefangenschaft und Psychosen», Der Nervenarzt 22 (1951) 149f.

Schützwohl, Matthias, «Frühinterventionen nach traumatisierenden Erfahrungen», Fortschritte der Neurologie, Psychiatrie 68 (2000) 423-430

Schultz, J. H., «Diskussionsbeitrag zur Arbeit von W. v. Baeyer: «Erschöpfung und Erschöpftsein»», Der Nervenarzt 32 (1961) 467

Seifert, «Ueber nervöse Unfallerkrankungen und ihre forensische Bedeutung. Referat, 50. Sitzung der forensisch-psychiatrischen Vereinigung zu Dresden am 16. November 1899», Allgemeine Zeitschrift für Psychiatrie und psychisch-gerichtliche Medizin 57 (1900) 763f.

- «Über einen Fall von Unfallhysterie mit cutaner und sensorischer Anästhesie», Archiv für Psychiatrie und Nervenkrankheiten 37 (1903) 1054-1056

Siegrist, Johannes, «Die Bedeutung von Lebensereignissen für die Entstehung körperlicher und psychosomatischer Erkrankungen», Der Nervenarzt 51 (1980) 313-320

Sneshewski, A. W., «Die psychogenen Psychosen», Psychiatrie, Neurologie und medizinische Psychologie 7 (1955) 128

Soyka, Dieter, «Hirnatrophische Defektzustände nach Dystrophie und ihre Pathogenese», Der Nervenarzt 29 (1958) 347-354

Stern, Felix, «Über die akuten Situationspsychosen der Kriminellen», Allgemeine Zeitschrift für Psychiatrie und psychisch-gerichtliche Medizin 69 (1912) 764f.

- «Beitrag zur Klinik hysterischer Situationspsychosen», Archiv für Psychiatrie und Nervenkrankheiten 50 (1913) 640-787

Stiefler, Georg, «Begutachtungsfragen: Die sogenannte Unfallsneurose», Fortschritte der Neurologie und Psychiatrie und ihrer Grenzgebiete 1 (1929) 544-557

- «Begutachtungsfragen. Die sogenannte Unfallneurose II», Fortschritte der Neurologie und Psychiatrie und ihrer Grenzgebiete 3 (1931) 461-490

Strasser, F., «Zur Nosologie und Psychodynamik der Rentenneurose», Der Nervenarzt 45 (1974) $225-232$

Strauss, Hans, «Besonderheiten der nichtpsychotischen seelischen Störungen bei Opfern der nationalsozialistischen Verfolgung und ihre Bedeutung bei der Begutachtung», Der Nervenarzt 28 (1957) 344-350

Strömgren, Eric, «Psychogene Psychosen», Der Nervenarzt 57 (1986) 88-95 
Suckow, J., «Zur Symptomatologie der Traumatischen Psychosen», Psychiatrie, Neurologie und medizinische Psychologie 6 (1954) 343

Teusch, L., «Ein katamnestischer Nachtrag zur Rentenneurosenstudie», Fortschritte der Neurologie, Psychiatrie 52 (1984) 113-115

- /M.-P. Engelmeier, «Eine empirische Studie zur Frage der Rentenneurose», Fortschritte der Neurologie, Psychiatrie 50 (1982) 207-214

Tölle, Reiner, «Verfolgungserleben im nationalsozialistischen Alltag», Fortschritte der Neurologie, Psychiatrie 67 (1999) 348-358

Trautmann, Edgar C., «Psychiatrische Untersuchungen an Überlebenden der nationalsozialistischen Vernichtungslager 15 Jahre nach der Befreiung», Der Nervenarzt 32 (1961) 545-551

Umpfenbach, F., «Funktionelle Psychosen», in: Otto Snell (Hrsg.), Bericht über die psychiatrische Literatur im Jahre 1916. Literaturheft zu Band 74, Allgemeine Zeitschrift für Psychiatrie und psychisch-gerichtliche Medizin (Berlin 1919a) 1-29

- «Funktionelle Psychosen», in: Otto Snell (Hrsg.), Bericht über die psychiatrische Literatur im Jahre 1917. II. Literaturheft zu Band 75, Allgemeine Zeitschrift für Psychiatrie und psychischgerichtliche Medizin (Berlin 1919b) 107-132

Uschakov, G. K., «Material zur Untersuchung der Ätiogenese der Psychosen», Psychiatrie, Neurologie und medizinische Psychologie 11 (1959) 257

Venzlaff, Ulrich, «Erlebnisreaktiver Persönlichkeitswandel? Anmerkungen zum Aufsatz von R. Luthe: «Erlebnisreaktiver Persönlichkeitswandel〉 als Begriff der Begutachtung im Entschädigungrecht [Der Nervenarzt 39, 1968, 465]», Der Nervenarzt 40 (1969) 539-542

von der Stein, B./K. Podoll/B. Greve/K. Heinrich, «Kumulatives Trauma durch fortgesetzte sadistische Kindesmißhandlung bei einer Patientin mit chronischer Artefakt-Krankheit», Fortschritte der Neurologie, Psychiatrie 60 (1992) 119-125

Weber, «Traumatische Psychosen», Allgemeine Zeitschrift für Psychiatrie und psychischgerichtliche Medizin 62 (1905) 641f.

Weber, M. M./I. A. Antonijevic/T. Bronisch, «Die versorgungsrechtliche Beurteilung einer Posttraumatischen Belastungsstörung», Der Nervenarzt 69 (1998) 811-814

Wegener, Hermann, «Zur Psychologie der seelischen Überforderung», Der Nervenarzt 24 (1953) $137-143$

Wende, J., «Ein Fall von traumatischer Psychose», Allgemeine Zeitschrift für Psychiatrie und psychisch-gerichtliche Medizin 61 (1904) 296-311

Wieser, Stefan, «Psychische Überforderungsreaktionen. I. Monotonie und Stress», Archiv für Psychiatrie und Nervenkrankheiten vereinigt mit Zeitschrift für die gesamte Neurologie und Psychiatrie 203 (1962) 452-461

- «Psychische Überforderungsreaktionen:V.Zur Periodizität abnormer Erlebnisreaktionen», Archiv für Psychiatrie und Nervenkrankheiten vereinigt mit Zeitschrift für die gesamte Neurologie und Psychiatrie 206 (1964) 96-112

- IA. Sausmikat/F. K. Jungklaass, «Psychische Überforderungsreaktionen. II. Wesen der psychischen Sättigung und ihr Verlauf bei Konstitutionstypen», Archiv für Psychiatrie und Nervenkrankheiten vereinigt mit Zeitschrift für die gesamte Neurologie und Psychiatrie 203 (1962) 462-482

Wilmanns, Karl, «Gefängnispsychosen», Allgemeine Zeitschrift für Psychiatrie und psychischgerichtliche Medizin 65 (1908) 378-380

- «Statistische Untersuchungen über Haftpsychosen», Allgemeine Zeitschrift für Psychiatrie und psychisch-gerichtliche Medizin 67 (1910) 847-866

Windgassen, Klaus, «Bemerkungen zur Arbeit von E. Strömgren. Psychogene Psychosen», Der Nervenarzt 57 (1986) 616

Winscheid, Franz, «Über die klinischen Eigenthümlichkeiten der Unfallneurose nebst Bemerkungen über die Erfahrungen in Bezug auf Beobachtung und Behandlung von Unfallhysterikern im «Hermann-Haus» in Stötteritz bei Leipzig, Unfallnervenklinik der Sächsischen Baugewerks-Berufsgenossenschaft», Archiv für Psychiatrie und Nervenkrankheiten 43 (1908) 1329-1342

Witter, Hermann, «Erlebnisbedingte Schädigung durch Verfolgung», Der Nervenarzt 33 (1962) 509 .

Wöller, Wolfgang, «Traumawiederholung und Reviktimisierung nach körperlicher und sexueller Traumatisierung», Fortschritte der Neurologie, Psychiatrie 73 (2005) 83-90 